\title{
MICROPHYTOPLANKTON BIOMASS AND TROPHIC STATE OF THE ESTUARINE REGION OF SÃO FRANCISCO RIVER (NORTHEASTERN BRAZIL)
}

\author{
MELO-MAGALHÃES, E.M. ${ }^{1 *}$; MOURA, A.N. ${ }^{2}$; MEDEIROS, P.R.P. ${ }^{1}$ \& KOENING,M.L. ${ }^{3}$ \\ 1. Universidade Federal de Alagoas-UFAL, Instituto de Ciências Biológicas e da Saúde-ICBS, \\ Laboratórios Integrados de Ciência do Mar e Naturais-LABMAR, Maceió,AL, Brasil \\ 2. Universidade Federal Rural de Pernambuco-UFRPE, Programa de Pós-graduação em Botânica, \\ Recife,PE,Brasil \\ 3. Universidade Federal de Pernambuco-UFPE, Programa de Pós-graduação em Oceanografia. \\ Recife,PE, Brasil. \\ *Corresponding author: enaidemmm@gmail.com
}

\begin{abstract}
Melo-Magalhães, E.M.; Moura, A.N.; Medeiros, P.R.P. \& Koening, M.L., 2016. Microphytoplankton biomass and trophic state of the estuarine region of São Francisco river (northeastern Brazil). Braz. J. Aquat. Sci. Technol. 20(2). eISSN 1983-9057. DOI: 10.14210/bjast.v20n2. One of the most serious problems that affects estuaries is the excessive enrichment of nutrients, especially nitrogen and phosphorus compounds that result in increased productivity. Studies to evaluate the trophic conditions of the São Francisco River estuary were conducted at four stations in the rainy (July/ 2006 ) and dry (January/2007) seasons in neap and spring tides, during high tide and low tide. Microphytoplankton was collected using plankton nets with mesh size of $45 \mu \mathrm{m}$ and the samples for analysis of nutrients and Phytoplankton biomass (chlorophyll a) were obtained from the sub-surface layer with wide-mouthed plastic bottles. The waters of this ecosystem showed salinities ranging from an oligohaline to a limnetic regime, alkaline $\mathrm{pH}$ and water clarity ranging between $1.00 \mathrm{~m}$ and $2.60 \mathrm{~m}$. The concentrations of dissolved nutrients varied considerably, being highest during the dry season period. The Microphytoplankton was composed of 176 taxa, mainly distributed among the Chlorophyta, Charophyta and Bacillariophyta. Phytoplankton biomass (chlorophyll a) showed no significant differences between seasons, tides and tidal regimes, however the density (Cel..-1) was significantly higher in the dry season. Values of a TSI showed oligotrophic conditions in the four stations and in the two periods analyzed. In the dry season there was a higher cell density and increased levels of dissolved nutrients with the exception of nitrate that was considered higher in the rainy season. The Microphytoplankton species are characteristic of waters of low to moderate trophic level and the TSI indicated a predominance of oligotrophic conditions in the two seasons in the study area.
\end{abstract}

Keywords: Chlorophyll a, Estuary, Hydrology, Phytoplankton, Seasonality.

\section{INTRODUCTION}

Coastal regions are susceptible to general changes, determined by the action of tides, winds, rainfall, river discharge and other contributions from human activities, among other factors. Therefore, these environments show wide variability of hydrological characteristics, and are frequently highly productive systems (Melo-Magalhães et al., 2008; Hartmann \& Schettini, 1991).

The São Francisco River is an important Brazilian river, which, due to its multiple utilities, has been widely explored throughout its extension for power generation, irrigation, and public supply, besides serving as a receiver of organic and inorganic waste from different sources. Its mouth is located between the states of Alagoas and Sergipe, forming an estuarine environment, which is characterized according to the classification proposed by Pritchard (1955) as a partially stratified salt-wedge estuary or a vertically mixed estuary with almost uniform flow.
Estuaries are coastal environments in the transition zone between the continent and adjacent ocean, where seawater is diluted by freshwater from continental drainage. They are the only aquatic systems where there is dynamic interaction between freshwater, marine water, the terrestrial system and the atmosphere (Day Jr et al., 1989; Miranda et al., 2002).

In these environments, studies involving Microphytoplankton and trophic state assessment are valuable tools for understanding the water quality as it contributes to our knowledge of the biological response to increasing nutrients, especially phosphorus and nitrogen.

Regarding hydrological and phytoplankton studies in the São Francisco estuary, there's only record of the study by Eskinazi-Leça (1967/1969), Souza (1999), Mafalda-Júnior (2002), Knoppers et al. (2005), Medeiros et al. (2007; 2011), Melo-Magalhães at al. (2011). This study aimed to determine the biomass (chlorophyll a), density of Microphytoplankton and the trophic state of the estuary of the São Francisco river 
- Piaçabuçu, state of Alagoas - in the dry and rainy seasons.

\section{MATERIALS AND METHODS}

The São Francisco River is born in the south at about $1800 \mathrm{~m}$ altitude in the Canastra mountain range in the State of Minas Gerais, and the river flows into the SW Atlantic Ocean at the border between the States of Sergipe and Alagoas, NE Brazil. The river is $2,863 \mathrm{Km}$ long, and its hydrographic basin covers $639,219 \mathrm{Km}^{2}$ and corresponds to the most extensive basin within the Brazilian territory. Due to its length, the basin traverses several climatic regimes and comprises several physiographic compartments. It is traditionally divided into the Upper, Middle, Sub-Middle and Lower SFR sector (Medeiros et al., 2011). The São Francisco river and its coastal zone have been impacted by dam cascades constructed between the seventies and the nineties in the middle-lower sector of the basin.

The estuary is composed of an internal channel up to $12 \mathrm{~m}$ deep, one subaqueous bar perpendicular to the coast at the updrift side, and another arch near to parallel to the coast along the downdrift side and fits in the category of a delta-estuarine. The estuarinedelta of the São Francisco River lies within the direct pathway of the tropical oligotrophic South Equatorial Current. In the Estuarine area, a salt wedge may intrude up to a maximum of $10 \mathrm{~km}$ into the river mouth and maintain oligohaline conditions of surface waters (Knoppers et al., 2005).

Samples were collected at four stations located in the municipality of Piaçabuçu, state of Alagoas (10 $24^{\prime} 06^{\prime} S^{\prime}-36^{\circ} 26^{\prime} 34^{\prime \prime} \mathrm{W}$ and $10^{\circ} 29^{\prime} 52^{\prime} S^{\prime}$ $36^{\circ} 23^{\prime} 42^{\prime \prime} \mathrm{W}$ ) in July 2006 (rainy season) and January 2007 (dry season), Figure 1.
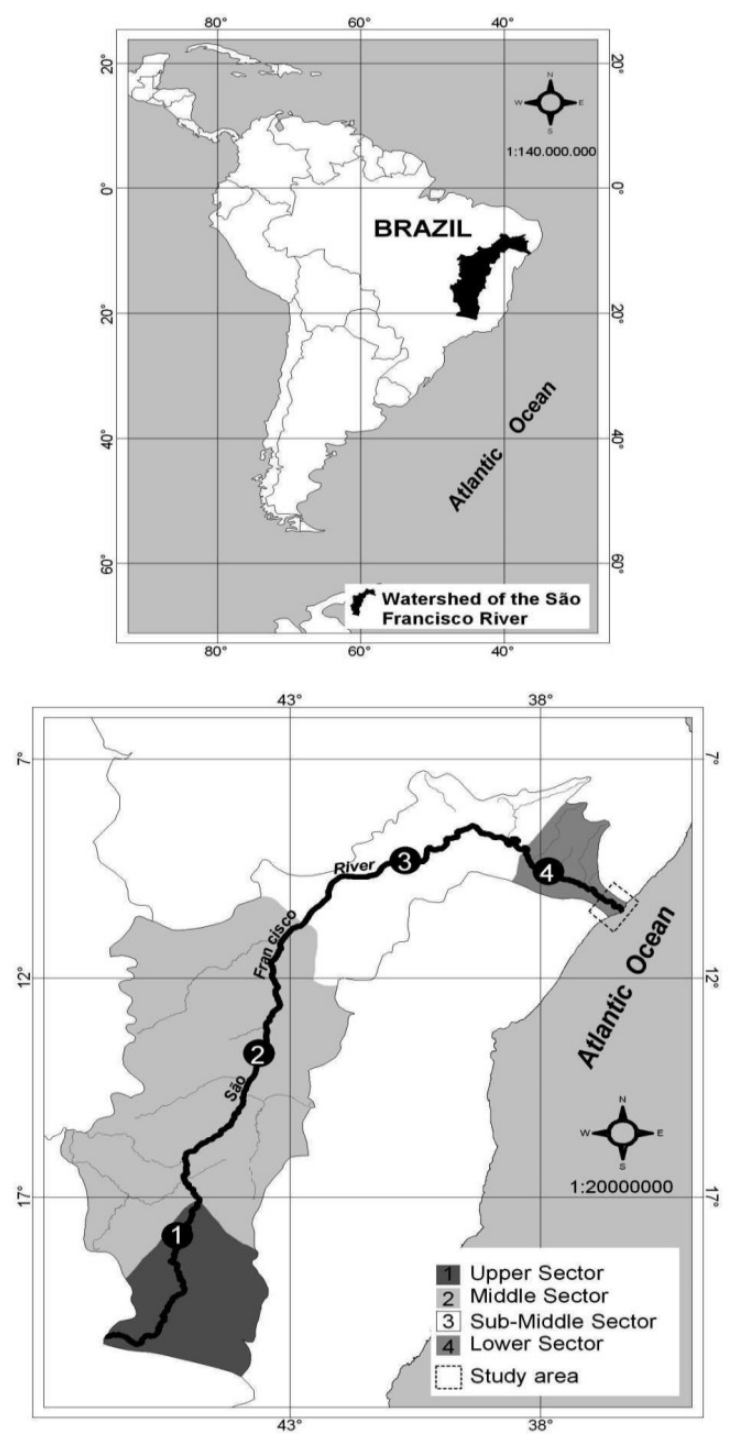

Figure 1 - Location of the sampling stations in the São Francisco River estuary, northeastern Brazil.

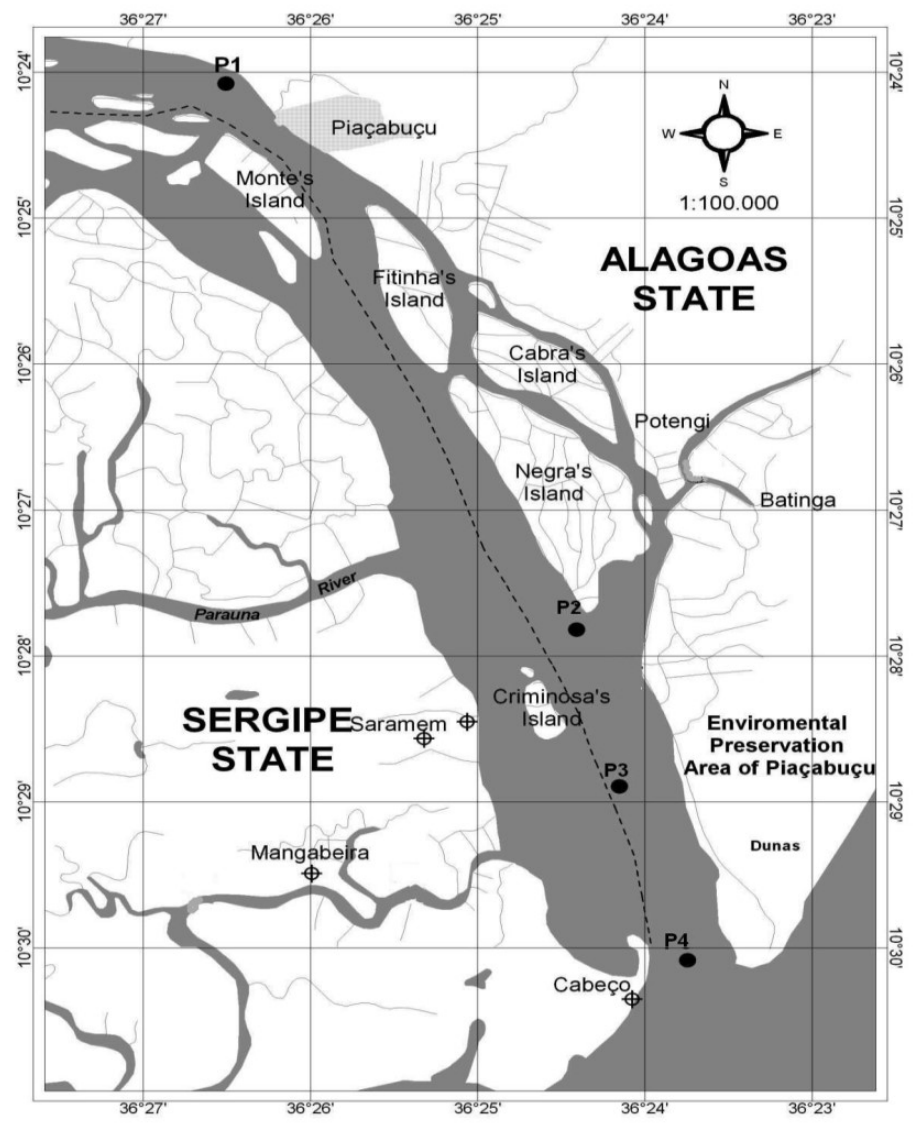


Samples of Microphytoplankton and abiotic data were collected at neap and spring tides, and during high tide and low tide in the subsurface layer. We used a Van Dorn bottle to study the chlorophyll $a$ and physicchemical parameter and plankton nets with a mesh opening of $45 \mu \mathrm{m}$ to collect the Microphytoplankton. Horizontal hauls were made with towing speed of approximately 1 knot, for five minutes.

Measurements of salinity, electrical conductivity, temperature, dissolved oxygen, dissolved oxygen saturation rate, $\mathrm{pH}$, turbidity and chlorophyll a were performed in situ with the aid of the multiparametric probe YSI 6600. Water transparency was measured using a Secchi disk.

Nitrite $\left(\mathrm{NO}^{2-}\right)$ nitrate $\left(\mathrm{NO}^{3-}\right)$ and ammonium $\left(\mathrm{NH}^{4+}\right)$ were determined by the method described in Strickland \& Parsons (1972), and silicon $\left(\mathrm{SiO}^{2-}\right)$, phosphorus $\left(\mathrm{PO}_{4}^{-2}\right)$ and dissolved phosphorus $\left(\mathrm{PO}_{4}^{-3}\right)$, according to Grasshoff et al. (1983). The Jenway 6.100 spectrophotometer was used for these analysis. Values were calculated from the ratio between the concentration of inorganic nitrogen (nitrate, nitrite, ammonium) and dissolved phosphorus (N:P), between the concentration of silicon and nitrogenous nutrients (Si:N), and between silicon and phosphate (Si:P). The atomic ratio of $16 \mathrm{~N}: 15 \mathrm{Si}: 1 \mathrm{P}$ was considered optimal for absorption of Microphytoplankton (Redfield et al., 1963).

The climatological data were obtained from meteorological stations Pão de Açúcar, Piranhas and Traipu, inserted in the low São Francisco Zone in Alagoas, located closest to the study area.

The trophic State Index (TSI) was accessed using Carlson (1977) modified by Toledo Jr et al. (1983) and Toledo (1990), for tropical ecosystems, calculated using the equations:

\begin{tabular}{ll}
\hline STI (med) & STI (PT)+STI $(\mathrm{PO} 4)+\mathrm{STI}(\mathrm{CL}) / 3$ \\
Dissolved Phosphorus & $\mathrm{STI}(\mathrm{PO})=10\left\{6-\left[\ln \left(21,67 / \mathrm{PO}_{4}\right) / \ln 2\right]\right\}$ \\
Total Phosphorus & $\mathrm{STI}(\mathrm{PT})=10\{6-[\ln (80,32 / \mathrm{PT}) / \ln 2]\}$ \\
Chlorophyll a & $\mathrm{STI}(\mathrm{CL})=10\{6-[(2,04-0,695 \ln \mathrm{CL}) / \ln 2]\}$ \\
\hline Where: $\mathrm{PT}, \mathrm{PO}_{4}, \mathrm{CL}\left(\mu \mathrm{g} . \mathrm{L}^{-1}\right) ;$ & In= natural logarithm
\end{tabular}

In order to analyze and identify the microphytoplankton species, $0.5 \mathrm{~mL}$ samples were examined in a Leica Galen III binocular microscope at zooms of 100x and 400x. The identification was made to the lowest possible taxonomic level and was based on specific literature, such as, Péragallo \& Péragallo (1897-1908), Hustedt (1930), Mizuno (1968), Prescott (1978), Anagnostidis \& Komárek (1990), Round et al. (1990), Moreno et al. (1996), Tomas (1997). To categorize the species, the classification system of the international Algaebase database was used (Guiry \& Guiry, 2016).

The Microphytoplanktonic density was calculated from samples collected on the surface and calculations were made from the filtered volume. Calculations from the filtered volume were made using the equation: $V=$ $r^{2}$.v.t, where $r^{2}$ is area plankton nets $\left(m^{2}\right)$; $v$ is towing speed $(\mathrm{m} / \mathrm{s})$ and $t$ is time to hauls(s). The filtered volume was $\left(10,82 \mathrm{~m}^{3}\right)$.

For statistical analysis (biotic and abiotic parameters), we used an analysis of variance (ANOVA) to detect the existence of significant differences between seasons (rainy and dry), tide regimes (neap and spring tides) and between tides (low tide and high tide). The t-test LSD was used to point out differences between the means of the treatments the biotic parameters (Zar, 1996). For the abiotic parameters was used the Tukey test (Cochran \& Cox, 1957).

\section{RESULTS}

Data obtained from the historical mean rainfall in areas close to the São Francisco River estuary (Figure 2a), showed a well-defined seasonal pattern, with two distinct periods: a rainy season (April to August) and a dry season (September to March). The average monthly rainfall (July/2006) at stations located in Pão de Açúcar, Piranhas and Traipú was, respectively, $178.70 \mathrm{~mm}, 154.40 \mathrm{~mm}$ and $148.4 \mathrm{~mm}$. In January/ 2007 (dry month), the average was $3.5 \mathrm{~mm}, 0.0 \mathrm{~mm}$ and $1.4 \mathrm{~mm}$, respectively (Figure $2 \mathrm{~b}$ ).

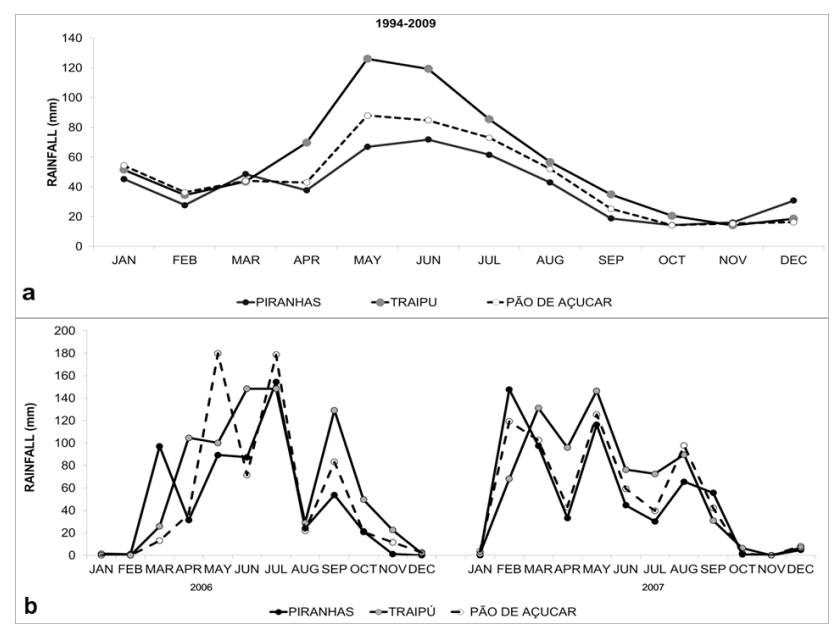

Figure 2 - Monthly rainfall at the Pão de Açucar, Piranhas and Traipú stations, state of Alagoas. a) Data from 1994 to 2009 and b) Data 2006 and 2007. Source: SEMARHN/AL.

Water transparency, turbidity, water temperature, $\mathrm{pH}$, dissolved oxygen, saturation rate, salinity values and electrical conductivity are shown in Table 1.

Significant differences between seasons were found with regards to water transparency, turbidity, $\mathrm{pH}$, water temperature, salinity and electrical conductivity. Water transparency, temperature, $\mathrm{pH}$ and electrical conductivity were higher in the dry season, although 
turbidity and salinity were higher in the rainy season. Significant differences between tides were found for water transparency, turbidity, water temperature, salinity and electrical conductivity. Significant difference between tide regime was found with regards only to $\mathrm{pH}$ (Table 2).

Table 1 - Abiotic data from the São Francisco River estuary during the rainy season (July 2006) and dry season (January 2007). LT = low tide, $\mathrm{HT}=$ high tide, SS 1-4 sampling stations.

\begin{tabular}{|c|c|c|c|c|c|c|c|c|}
\hline $\begin{array}{l}\text { Variation/ } \\
\text { Season }\end{array}$ & $\begin{array}{c}\text { Water } \\
\text { Transparency } \\
\text { (m) }\end{array}$ & Turbidity & $\begin{array}{c}\text { Temperature } \\
\left({ }^{\circ} \mathrm{C}\right)\end{array}$ & $\mathrm{pH}$ & $\begin{array}{c}\text { Dissolved } \\
\text { Oxygen } \\
(\mathrm{mg} / \mathrm{L}) \\
\end{array}$ & $\begin{array}{c}\text { Saturation } \\
\text { rate } \\
(\%)\end{array}$ & Salinity & $\begin{array}{c}\text { Electrical } \\
\text { Condutivity } \\
(1 \mathrm{~S} / \mathrm{cm})\end{array}$ \\
\hline \multicolumn{9}{|c|}{ Rainy Season (July 2006) } \\
\hline \multicolumn{9}{|c|}{ Neap Tide } \\
\hline SS1-LT & 1.70 & 13.50 & 25.67 & 7.65 & 7.91 & 97.00 & 0.00 & 171.50 \\
\hline SS2-LT & 1.60 & 10.70 & 26.18 & 7.54 & 7.64 & 94.50 & 0.08 & 173.00 \\
\hline SS3-LT & 1.60 & 9.50 & 25.83 & 7.54 & 7.90 & 97.00 & 0.07 & 155.00 \\
\hline SS4-LT & 1.70 & 9.50 & 25.90 & 7.52 & 7.61 & 93.60 & 0.06 & 134.00 \\
\hline SS1-HT & 2.00 & 7.00 & 25.30 & 7.54 & 7.61 & 92.70 & 0.06 & 133.00 \\
\hline SS2-HT & 1.60 & 17.00 & 25.97 & 7.50 & 7.48 & 92.20 & 0.08 & 159.00 \\
\hline SS3-HT & 1.90 & 7.50 & 26.16 & 7.36 & 7.59 & 93.90 & 0.08 & 159.00 \\
\hline SS4-HT & 1.60 & 9.00 & 25.98 & 7.32 & 7.89 & 97.20 & 0.07 & 158.00 \\
\hline Mean & 1.71 & 10.46 & 25.87 & 7.50 & 7.70 & 94.76 & 0.06 & 155.31 \\
\hline \multicolumn{9}{|c|}{ Spring Tide } \\
\hline SS1-LT & 1.00 & 19.60 & 25.32 & 7.29 & 7.60 & 92.40 & 0.10 & 220.00 \\
\hline SS2-LT & 1.00 & 16.70 & 25.31 & 7.40 & 7.39 & 90.10 & 0.10 & 219.00 \\
\hline SS3-LT & 1.20 & 16.20 & 25.38 & 7.54 & 7.73 & 94.20 & 0.09 & 203.00 \\
\hline SS4-LT & 1.40 & 13.60 & 25.40 & 7.55 & 7.78 & 94.90 & 0.08 & 173.00 \\
\hline SS1-HT & 1.30 & 12.60 & 25.44 & 7.50 & 7.51 & 91.80 & 0.12 & 253.00 \\
\hline SS2-HT & 1.30 & 15.80 & 25.81 & 7.67 & 7.79 & 95.60 & 0.10 & 217.00 \\
\hline SS3-HT & 1.30 & 16.50 & 25.59 & 7.59 & 7.61 & 93.00 & 0.12 & 252.00 \\
\hline SS4-HT & 1.10 & 21.70 & 25.96 & 7.63 & 8.90 & 99.00 & 0.23 & 482.00 \\
\hline Mean & 1.20 & 16.59 & 25.53 & 7.52 & 7.80 & 93.88 & 0.12 & 252.38 \\
\hline \multicolumn{9}{|c|}{ Dry Season (January 2007). } \\
\hline \multicolumn{9}{|c|}{ Neap Tide } \\
\hline SS1-- LT & 2.10 & 6.30 & 28.65 & 7.75 & 7.91 & 100.00 & 0.00 & 76.00 \\
\hline SS2-- LT & 2.10 & 4.70 & 28.57 & 7.42 & 7.33 & 94.60 & 0.00 & 80.00 \\
\hline SS3-- LT & 2.00 & 6.10 & 28.43 & 7.40 & 8.15 & 104.90 & 0.00 & 93.00 \\
\hline SS4-- LT & 2.40 & 4.20 & 28.34 & 7.38 & 7.39 & 95.00 & 0.00 & 93.00 \\
\hline SS1-HT & 2.10 & 6.30 & 28.65 & 7.75 & 7.91 & 100.00 & 0.00 & 76.00 \\
\hline SS2-HT & 2.40 & 4.70 & 28.51 & 7.75 & 6.39 & 82.30 & 0.00 & 82.00 \\
\hline SS3-HT & 2.60 & 4.10 & 28.48 & 7.81 & 7.57 & 100.30 & 0.00 & 92.00 \\
\hline SS4-HT & 2.60 & 3.50 & 28.35 & 7.62 & 7.82 & 100.00 & 0.00 & 133.00 \\
\hline Mean & 2.29 & 4.99 & 28.50 & 7.61 & 7.68 & 97.14 & 0.00 & 90.63 \\
\hline \multicolumn{9}{|c|}{ Spring Tide } \\
\hline SS1- LT & 2.60 & 8.10 & 28.55 & 7.69 & 7.60 & 98.00 & 0.00 & 74.00 \\
\hline SS2- LT & 2.60 & 5.50 & 28.41 & 7.68 & 7.84 & 99.00 & 0.00 & 121.00 \\
\hline SS3- LT & 2.50 & 9.20 & 28.36 & 7.89 & 8.10 & 100.00 & 0.00 & 103.00 \\
\hline SS4- LT & 2.40 & 6.80 & 28.30 & 7.88 & 8.10 & 101.00 & 0.00 & 205.00 \\
\hline SS1-HT & 2.60 & 8.10 & 28.55 & 7.69 & 7.60 & 98.00 & 0.00 & 74.00 \\
\hline SS2- HT & 2.20 & 6.20 & 28.52 & 7.55 & 7.83 & 99.00 & 0.00 & 76.00 \\
\hline SS3-HT & 2.30 & 5.60 & 28.71 & 7.73 & 8.00 & 101.00 & 0.00 & 77.00 \\
\hline SS4-HT & 2.10 & 9.80 & 28.63 & 7.49 & 8.10 & 100.00 & 0.00 & 116.00 \\
\hline Mean & 2.41 & 7.41 & 28.50 & 7.70 & 7.90 & 99.50 & 0.00 & 105.75 \\
\hline
\end{tabular}

The ANOVA revealed significant differences between interactions: season with tide for water transparency, turbidity, water temperature and between interactions: season with tide regime for $\mathrm{pH}$ (Table 2).
The concentrations of nitrogenous elements, dissolved phosphorus, total phosphorus, silicon, N:P ratio, Si:N and Si:P values are shown in Table 3. 
Table 2 - Values (F) tested by ANOVA for the abiotic and biological data from the São Francisco River estuary during the rainy season (July 2006) and dry season (January 2007).

\begin{tabular}{|c|c|c|c|c|c|}
\hline Sources of Variation & A- Season & B-Tides & $\begin{array}{l}\text { C-Tidal } \\
\text { Regimes }\end{array}$ & $A \times B$ & $A \times C$ \\
\hline Transparency & $217.04^{\star *}$ & $12.84^{* *}$ & 5.71. & $19.18^{\star *}$. & 0.16 \\
\hline Turbidity & $68.09^{* *}$ & $23.19^{* *}$ & 0.12 & $4.34^{*}$ & 0.00 \\
\hline $\mathrm{pH}$ & $11.58^{* *}$ & 1.79 & 6.41* & 0.57 & $5.28^{*}$ \\
\hline Temperature & $1508.25^{\star *}$ & $5 . .60^{*}$ & 3.04 & $6.02^{*}$ & 0.14 \\
\hline Dissolved oxygen & $0.02 \mathrm{~ns}$ & $2.27 \mathrm{~ns}$ & $0.01 \mathrm{~ns}$ & $0.81 \mathrm{~ns}$ & $0.65 \mathrm{~ns}$ \\
\hline Salinity & $106.63^{\star *}$ & $9.56^{\star \star}$ & 4.03 & $9.96^{* *}$ & 4.03 \\
\hline Electrical conductivity & $32.26^{\star *}$ & $9.14^{\star \star}$ & 3.64 . & $4.88^{\star}$ & 0.31 \\
\hline Ammonium & $13.71^{\star \star}$ & $0.01 \mathrm{~ns}$ & $0.21 \mathrm{~ns}$ & $0.92 \mathrm{~ns}$ & $0.00 \mathrm{~ns}$ \\
\hline Nitrite & $0.81 \mathrm{~ns}$ & $2.07 \mathrm{~ns}$ & $0.56 \mathrm{~ns}$ & $0.68 \mathrm{~ns}$ & $1.16 \mathrm{~ns}$ \\
\hline Nitrate & $42.58^{* *}$ & $0.03 \mathrm{~ns}$ & $0.01 \mathrm{~ns}$ & $0.41 \mathrm{~ns}$ & $0.04 \mathrm{~ns}$ \\
\hline Dissolved phosphorus & $177.20^{\star *}$ & $0.00 \mathrm{~ns}$ & $2.77 \mathrm{~ns}$ & $1.50 \mathrm{~ns}$ & $3.67 \mathrm{~ns}$ \\
\hline Total phosphorus & $788.32^{\star *}$ & $0.02 \mathrm{~ns}$ & $0.10 \mathrm{~ns}$ & $0.40 \mathrm{~ns}$ & $1.97 \mathrm{~ns}$ \\
\hline Silicon & $3.08 \mathrm{~ns}$ & $1.07 \mathrm{~ns}$ & $0.16 \mathrm{~ns}$ & $3.75 \mathrm{~ns}$ & $0.58 \mathrm{~ns}$ \\
\hline Chlorophyll a & $3.29 \mathrm{~ns}$ & $0.00 \mathrm{~ns}$ & $1.64 \mathrm{~ns}$ & $2.56 \mathrm{~ns}$ & $2.77 \mathrm{~ns}$ \\
\hline Total Density & 10.56 * & $1.035 \mathrm{~ns}$ & $1.487 \mathrm{~ns}$ & $0.680 \mathrm{~ns}$ & $0.254 \mathrm{~ns}$ \\
\hline
\end{tabular}

Table 3 - Mean values of dissolved nutrients and N/P, Si/N and Si/P ratios in the São Francisco River estuary during the rainy season (July 2006) and dry season (January 2007). SS 1-4-sampling stations; LT = low tide, HT = high tide.

\begin{tabular}{|c|c|c|c|c|c|c|c|c|c|}
\hline Nutrients & $\begin{array}{l}\mathrm{N}-\mathrm{NH} 3 \\
(\mu \mathrm{gN} / \mathrm{L})\end{array}$ & $\begin{array}{l}\mathrm{N}-\mathrm{NO} 2 \\
(\mu \mathrm{gN} / \mathrm{L})\end{array}$ & $\begin{array}{l}\mathrm{N}-\mathrm{NO} 3 \\
(\mu \mathrm{gN} / \mathrm{L}) \\
\end{array}$ & $\begin{array}{l}\mathrm{P}-\mathrm{PO} 4 \\
(\mu \mathrm{gP} / \mathrm{L}) \\
\end{array}$ & $\begin{array}{c}\mathrm{PT} \\
(\mu \mathrm{gP} / \mathrm{L}) \\
\end{array}$ & $\begin{array}{c}\text { Si (-SIO4) } \\
\mu \mathrm{gSi} / \mathrm{L}\end{array}$ & $\begin{array}{l}\text { N/P } \\
\text { Ratio }\end{array}$ & $\begin{array}{l}\text { Si/N } \\
\text { Ratio }\end{array}$ & $\begin{array}{l}\text { Si/P } \\
\text { Ratio }\end{array}$ \\
\hline \multicolumn{10}{|c|}{ Rainy Season (July 2006) } \\
\hline \multicolumn{10}{|c|}{ Neap Tide } \\
\hline SS1-LT & 16.80 & 2.52 & 96.18 & 1.86 & 2.52 & 3740.82 & 62.16 & 37.2 & 2013.14 \\
\hline SS2-LT & 5.60 & 2.38 & 69.16 & 1.86 & 5.88 & 4021.62 & 41.51 & 52.3 & 2164.25 \\
\hline SS3-LT & 2.24 & 3.36 & 35.42 & 3.72 & 6.44 & 4037.34 & 11.04 & 88.58 & 1086.36 \\
\hline SS4-LT & 9.52 & 4.34 & 68.04 & 2.48 & 3.92 & 3756.54 & 33.06 & 50.47 & 1516.2 \\
\hline SS1-HT & 16.10 & 2.24 & 95.2 & 1.86 & 2.66 & 3740.82 & 61.1 & 37.51 & 2013.14 \\
\hline SS2-HT & 5.04 & 12.32 & 15.68 & 2.48 & 2.80 & 4021.62 & 13.34 & 191.89 & 1623.19 \\
\hline SS3-HT & 10.08 & 7.98 & 54.60 & 2.40 & 1.68 & 3896.94 & 29.33 & 66.32 & 1572.87 \\
\hline SS4-HT & 14.14 & 2.52 & 74.34 & 1.86 & 2.52 & 3850.05 & 48.97 & 48.91 & 2071.92 \\
\hline Mean & & & & 2.32 & 3.55 & & 37.56 & 71.65 & 1757.64 \\
\hline \multicolumn{10}{|c|}{ Spring Tide } \\
\hline SS1-LT & 4.48 & 2.10 & 50.54 & 3.10 & 4.76 & 4411.65 & 18.44 & 75.55 & 1424.49 \\
\hline SS2-LT & 4.48 & 2.38 & 65.94 & 3.10 & 3.92 & 0.85 & 23.51 & 56.62 & 1333.82 \\
\hline SS3-LT & 31.92 & 2.52 & 117.18 & 3.10 & 2.80 & 19 & 48.96 & 34.07 & 1353.95 \\
\hline SS4-LT & 12.88 & 2.52 & 65.66 & 2.48 & 2.52 & 0.85 & 32.72 & 58.46 & 1667.28 \\
\hline SS1-HT & 17.36 & 18.20 & 105.00 & 2.48 & 3.08 & & 56.73 & 30.59 & 1365.01 \\
\hline SS2-HT & 17.36 & 2.10 & 55.30 & 2.48 & 3.64 & 2.02 & 30.17 & 67.77 & 1679.86 \\
\hline SS3-HT & 11.76 & 1.82 & 60.48 & 2.48 & 3.92 & 4255.80 & 29.89 & 63.64 & 1717.71 \\
\hline SS4-HT & 7.28 & 13.16 & 36.12 & 2.48 & 1.68 & 4286.97 & 22.83 & 106.44 & 1730.29 \\
\hline Mean & 13.44 & 5.60 & 69.53 & 2.71 & 3.29 & 4.119 .16 & 32.91 & 61.64 & 1.534 .05 \\
\hline \multicolumn{10}{|c|}{ Dry Season (January 2007) } \\
\hline \multicolumn{10}{|c|}{ Neap Tide } \\
\hline SS1-LT & 31.55 & 2.61 & 25.94 & 7.37 & 16.67 & 4.175 .37 & 8.15 & 83.55 & 566.24 \\
\hline SS2-LT & 16.48 & 2.61 & 18.09 & 5.90 & 18.67 & 4.114 .33 & 6.30 & 96.47 & 697.46 \\
\hline SS3-LT & 28.86 & 2.61 & 24.25 & 5.90 & 21.33 & 3.516 .10 & 9.45 & 68.29 & 596.05 \\
\hline SS4-LT & 21.86 & 1.12 & 17.89 & 4.42 & 18.00 & 3.650 .40 & 9.24 & 90.54 & 825.08 \\
\hline SS1-HT & 31.55 & 2.61 & 25.94 & 7.37 & 16.67 & 4.175 .37 & 8.15 & 83.55 & 566.24 \\
\hline SS2-HT & 19.71 & 2.23 & 18.64 & 7.37 & 20.00 & 3.699 .23 & 5.50 & 80.39 & 501.67 \\
\hline SS3-HT & 19.71 & 1.12 & 19.20 & 8.85 & 18.67 & 3.833 .53 & 4.52 & 82.06 & 433.24 \\
\hline SS4-HT & & 2.61 & 17.71 & 7.37 & 16.67 & 4.016 .66 & 5.28 & 96.20 & 544.72 \\
\hline Mean & 23.54 & 2.19 & 20.96 & 6.82 & 18.33 & 3.897 .63 & 7.07 & 85.13 & 591.34 \\
\hline \multicolumn{10}{|c|}{ Spring Tide } \\
\hline SS1-LT & 20.78 & 3.35 & 18.28 & 7.37 & 18.67 & 3.699 .23 & 5.75 & 83.47 & 501.67 \\
\hline SS2-LT & 1.40 & 12.29 & 25.04 & 7.37 & 18.00 & 3.943 .41 & 5.25 & 78.22 & 534.79 \\
\hline SS3-LT & 35.86 & 1.86 & 16.59 & 5.90 & 20.00 & 3.711 .44 & 9.21 & 87.36 & 629.16 \\
\hline SS4-LT & 27.25 & 6.70 & 17.54 & 5.90 & 17.33 & 3.931 .20 & 8.73 & 96.42 & 666.41 \\
\hline SS1-HT & 20.78 & 3.35 & 18.28 & 7.37 & 18.67 & 3.699 .23 & 5.75 & 83.47 & 501.67 \\
\hline SS2-HT & 13.78 & 3.72 & 17.72 & 7.37 & 22.00 & 3.833 .53 & 4.78 & 81.41 & 519.88 \\
\hline & & & & 4.42 & 18.00 & 3.857 .95 & 9.17 & 118.76 & 871.99 \\
\hline SS4-HT & 27.25 & 6.70 & 17.54 & 5.90 & 17.33 & 3.931 .20 & 8.73 & 96.42 & 666.41 \\
\hline Mean & 21.46 & 5.49 & 17.63 & 6.45 & 18.75 & 3.825 .90 & 7.17 & 90.69 & 611.50 \\
\hline
\end{tabular}


For dissolved nutrients the ANOVA revealed significant differences between the seasons for the elements: ammonium, nitrate, dissolved phosphorus and total phosphorus (Table 2). Ammonia, total phosphorus and dissolved phosphorus values were higher for the dry season, but nitrate was higher in the rainy season. Overall, taking into account the average values obtained at the neap and spring tides, we observed higher values for the N:P and Si:P ratios in the rainy season. The Si: $\mathrm{N}$ ratio presented higher values in the dry season.

Trophic State Index and values and Microphytoplankton biomass (Chlorophyll a) of the São Francisco River estuary are shown in Figures 3 and 4.

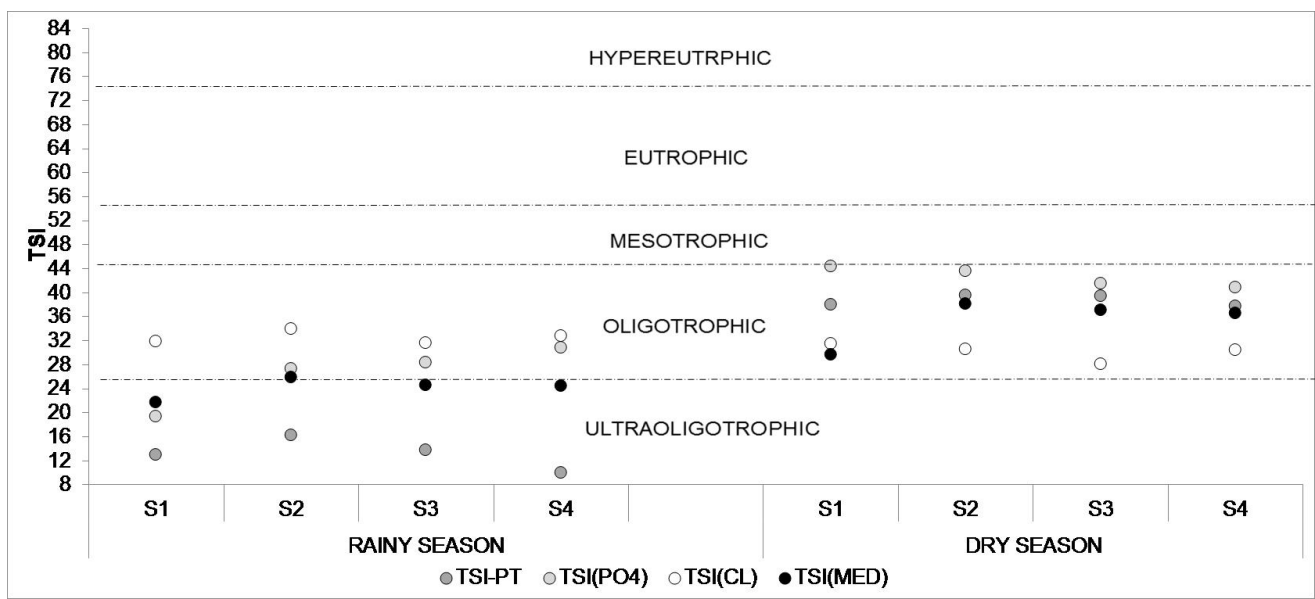

Figure 3 - Mean values of the trophic state index STI (mean), STI (PT), STI (PO $)$ and STI (CL) in the São Francisco River estuary during the rainy season (July 2006) and dry season (January 2007).

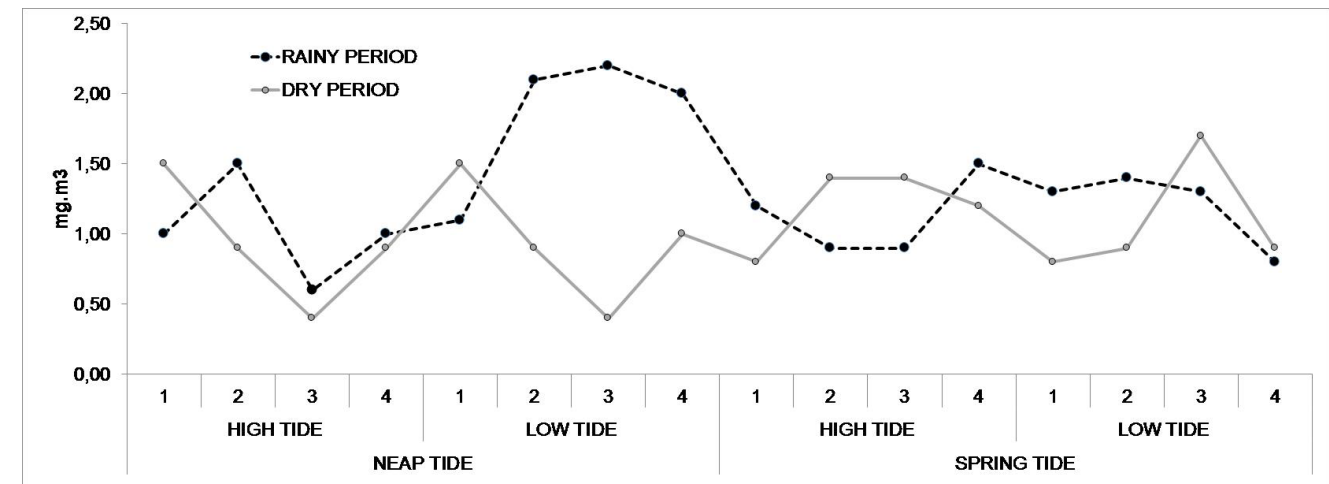

Figure 4 - Mean values of chlorophyll a in the São Francisco River estuary during the rainy season (July 2006) and dry season (January 2007). 1-4 sampling stations.

For chlorophyll a the ANOVA revealed no significant differences between seasons, Tides, Tide Regime, interactions season with tide and season with tide regime and for Microphytoplankton density, significant differences occurred between seasons (Table 2).

The Microphytoplankton was made up of 176 taxa, mainly distributed among the Chlorophyta, Charophyta and Bacillariophyta. The density in the São Francisco River estuary revealed a marked variation between seasons (rainy and dry). In the rainy season, the densities varied between $187 \mathrm{Cel}^{-\mathrm{L}^{-1}}$ and 5551 Cel. $\mathrm{L}^{-1}$ and in the dry season the densities were much higher and fluctuated between 901 Cel.L-1 and 29737
Org. $\mathrm{L}^{-1}$. Diatoms and green algae stood out due to their high density. The highest densities of these groups were observed during the dry season, at station S2, at high tide during spring tide where the following were recorded: 12384 Cel.L-1 (Bacillariophyta), 12431 Cel.L-1 (Chlorophyta) and 4876 Cel.L-1 (Charophyta). The other groups had a Microphytoplankton density below 195 Cel.L-1 (Table 4).

In the rainy season, the highest densities were recorded for the diatom Aulacoseira ambigua var. ambigua $f$. spiralis (Skuja) Ludwig especially at the neap tide. In the dry season, Fragilaria crotonensis Kitton and Pediastrum boryanum (Turpin) Meneghini (in the neap tide) stood out (Table 5). 
Braz. J. Aquat. Sci. Technol., 2016, 20(2).

Table 4 - Distribution of the Microphytoplankton density (Cel.L-1) groups in the São Francisco River estuary during the rainy season (July 2006) and dry season (January 2007). SS: sampling stations: 1-4 sampling stations.

\begin{tabular}{|c|c|c|c|c|c|c|c|c|}
\hline Tide & \multicolumn{4}{|c|}{ High tide } & \multicolumn{4}{|c|}{ Low tide } \\
\hline Divisions/Stations & SS1 & SS2 & SS3 & SS4 & SS1 & SS2 & SS3 & SS4 \\
\hline \multicolumn{9}{|c|}{ Rainy Season (July 2006) } \\
\hline \multicolumn{9}{|c|}{ Neap Tide } \\
\hline Cyanobacteria & 25 & 7 & 2 & 83 & 63 & 7 & 9 & 7 \\
\hline Miozoa & --- & 5 & --- & --- & --- & --- & --- & --- \\
\hline Euglenophyta & .-- & --- & -.- & 7 & --- & --- & --- & --- \\
\hline Bacillariophyta & 3532 & 2825 & 3869 & 3835 & 1780 & 892 & 1095 & 4231 \\
\hline Chlorophyta & 1535 & 962 & 1169 & 1359 & 257 & 301 & 692 & 1255 \\
\hline Charophyta & 51 & 73 & 48 & 111 & 56 & 39 & 56 & 58 \\
\hline Total $\left(\right.$ Cel. $\left.\mathrm{L}^{-1}\right)$ & 5143 & 3872 & 5088 & 5395 & 2156 & 1239 & 1852 & 5551 \\
\hline \multicolumn{9}{|c|}{$\begin{array}{l}\text { Spring Tide } \\
\end{array}$} \\
\hline Cyanobacteria & 14 & 47 & 19 & 2 & --- & 2 & --- & 4 \\
\hline Miozoa & --- & 5 & 5 & --- & --- & --- & --- & \\
\hline Bacillariophyta & 435 & 1581 & 971 & 1179 & 77 & 443 & 784 & 544 \\
\hline Chlorophyta & 233 & 1113 & 617 & 264 & 67 & 36 & 302 & 114 \\
\hline Charophyta & 28 & 144 & 103 & 39 & 43 & 2 & 86 & 11 \\
\hline Total $($ Cel.L-1) & 710 & 2890 & 1715 & 1484 & 187 & 483 & 1172 & 673 \\
\hline \multicolumn{9}{|c|}{ Dry season (January 2007) } \\
\hline \multicolumn{9}{|c|}{ Neap Tide } \\
\hline Cyanobacteria & 23 & 23 & 116 & 2 & 46 & 23 & 194 & 23 \\
\hline Euglenophyta & --- & 23 & --- & --- & -- & -- & --- & 23 \\
\hline Bacillariophyta & 1132 & 2056 & 8688 & 1213 & 1687 & 5545 & 4298 & 4506 \\
\hline Chlorophyta & 2221 & 2058 & 12616 & 1214 & 2801 & 5684 & 3326 & 4413 \\
\hline Charophyta & 691 & 369 & 1062 & 70 & 854 & 948 & 860 & 924 \\
\hline Total $($ Cel.L-1 $)$ & 4067 & 4529 & 22482 & 2499 & 5388 & 12200 & 8678 & 9889 \\
\hline \multicolumn{9}{|c|}{ Spring Tide } \\
\hline Cyanobacteria & 92 & 46 & 55 & --- & 195 & 18 & 166 & 14 \\
\hline Bacillariophyta & 9057 & 12384 & 7736 & 957 & 2275 & 1543 & 4270 & 347 \\
\hline Chlorophyta & 4366 & 12431 & 10123 & 693 & 2084 & 1387 & 4882 & 388 \\
\hline Charophyta & 1781 & 4876 & 2160 & 152 & 2382 & 369 & 1791 & 152 \\
\hline Total $\left(\right.$ Cel. $\left.\mathrm{L}^{-1}\right)$ & 15296 & 29737 & 20074 & 1802 & 6936 & 3317 & 11109 & 901 \\
\hline
\end{tabular}

Table 5 - Density $\left(\right.$ Cel. $\left.\mathrm{L}^{-1}\right)$ of the most representative taxa (> $\left.500 \mathrm{Cel} . \mathrm{L}^{-1}\right)$ at neap and spring tides (high tide and low tide), during the rainy season (July 2006) and dry season (January 2007); SS: sampling stations: 1-4 sampling stations.

\begin{tabular}{|c|c|c|c|c|c|c|c|c|}
\hline Tide & \multicolumn{4}{|c|}{ High tide } & \multicolumn{4}{|c|}{ Low tide } \\
\hline Taxa /Stations & SS1 & SS2 & SS3 & SS4 & SS1 & SS2 & SS3 & SS4 \\
\hline \multicolumn{9}{|c|}{ Rainy Season (July 2006) } \\
\hline \multicolumn{9}{|c|}{ Neap Tide } \\
\hline $\begin{array}{l}\text { Aulacoseira ambigua (Grunow) Simonsen } \\
\text { Aulacoseira ambigua var. ambigua f, spiralis }\end{array}$ & 28 & 134 & 469 & --- & 388 & 88 & 201 & 866 \\
\hline (Skuja) Ludwig & 2253 & 1811 & 2518 & 1622 & 1248 & 689 & 693 & 2662 \\
\hline Aulacoseira granulata (Ehrenberg) Simonsen & 1026 & 712 & 656 & 1920 & 97 & 65 & 201 & 451 \\
\hline Eudorina elegans Ehrenberg & 721 & 134 & 414 & 430 & 14 & 32 & 243 & 319 \\
\hline Pediastrum duplex Meyen & 652 & 462 & 92 & 693 & 7 & 14 & 14 & 111 \\
\hline \multicolumn{9}{|c|}{ Spring Tide } \\
\hline Aulacoseira granulata (Ehrenberg) Simonsen & --- & 961 & 330 & 543 & 28 & 92 & 444 & 437 \\
\hline Eudorina elegans Ehrenberg & 46 & 471 & 323 & 113 & 2 & --- & 104 & 42 \\
\hline \multicolumn{9}{|c|}{ Dry Season (January 2007) } \\
\hline \multicolumn{9}{|c|}{ Neap Tide } \\
\hline Microcystis aeruginosa (Kützing) Kützing & --- & 23 & --- & 2 & --- & --- & 111 & --- \\
\hline Aulacoseira granulata (Ehrenberg) Simonsen & 23 & 254 & 1155 & 23 & 208 & 901 & 277 & 601 \\
\hline Fragilaria crotonensis Kitton & 924 & 1594 & 6909 & 1171 & 1155 & 3443 & 3577 & 3697 \\
\hline Plourosira laevis (Ehrenberg) Compère & 23 & 69 & 46 & 2 & 23 & 555 & 83 & 23 \\
\hline Synedra sp. & 23 & 46 & 393 & 2 & 116 & 578 & 166 & 46 \\
\hline $\begin{array}{l}\text { Mucidosphaerium pulchellum (H.C.Wood) } \\
\text { C.Bock, Proschold \& Krienitz }\end{array}$ & 162 & 23 & 994 & 5 & 46 & 92 & 416 & 23 \\
\hline Pediastrum boryanum (Turpin) Meneghini & 1617 & 1294 & 10467 & 1157 & 2153 & 4644 & 2274 & 3396 \\
\hline \multicolumn{9}{|c|}{ Spring Tide } \\
\hline Aulacoseira granulata (Ehrenberg) Simonsen & 670 & 3350 & 1359 & $-\ldots$ & 28 & --- & 1091 & 14 \\
\hline Fragilaria crotonensis Kitton & 7278 & 7579 & 6100 & 679 & 970 & 1007 & 2754 & 236 \\
\hline Synedra sp. & 393 & 786 & 166 & 55 & 555 & 194 & --- & 55 \\
\hline Ulnaria ulna (Nitzsch) Ehrenberg & 439 & 92 & 28 & 14 & 222 & 55 & 185 & --- \\
\hline Desmidium baileyi (Ralfs) Nordstedt & 347 & 1086 & 55 & --- & 28 & --.- & --- & 14 \\
\hline Gonatozygon monotaenium De Bary & --- & -- & 610 & --- & 1913 & 28 & 444 & --- \\
\hline Gonatozygon sp. & 116 & 578 & --- & -.- & -.- & --- & --- & --- \\
\hline Monactinus simplex (Meyen) Corda & 232 & 370 & 804 & 14 & 111 & 1,257 & 37 & -- \\
\hline Pediastrum boryanum (Turpin) Meneghini & 3812 & 10998 & 8290 & 596 & 1885 & --- & 3789 & 347 \\
\hline Plourotaenium trabecula Nägeli & 162 & 508 & 388 & --- & 28 & -- & 203 & -- \\
\hline Sphaerocystis schroeteri Chodat & 23 & 323 & 915 & --- & --- & 28 & 74 & --- \\
\hline Spirogyra sp. & 46 & 555 & 665 & 14 & 166 & --- & 259 & --- \\
\hline Staurastrum rotula Nordstedt & 439 & 1040 & 388 & 55 & 28 & 157 & 314 & 42 \\
\hline
\end{tabular}




\section{DISCUSSION}

As in most estuarine ecosystems in Brazil, the São Francisco River estuary is a highly dynamic environment, with significant seasonal and spatial changes in its physical, chemical and biological parameters. This is mainly due to interactions between climatic and hydrological factors. For Miranda et al. (2002), estuaries are influenced by the action of climatic, oceanographic, geological, hydrological, biological and chemical events.

In tropical aquatic ecosystems, despite small variations in temperature and luminosity, seasonality is often observed with regards to the physical-chemical parameters and the Microphytoplankton community. Regarding water temperature, although lower during the rainy season, the values recorded in this study were consistently high, a range of $4.3^{\circ} \mathrm{C}$ in both periods. The small temperature variations observed in these environments are not likely to affect the growth and abundance of Microphytoplankton or determine the occurrence of an annual pattern (Agawin \& Duarte, 2002). Similar results were obtained by Souza et al. (1999) and Knoppers et al. (2005).

A seasonal pattern was not observed with regard to concentration of dissolved oxygen. The maximum value was $8.90 \mathrm{mg} / \mathrm{L}$ with a saturation rate of $99.00 \%$. Dissolved oxygen is considered an important indicator of polluted areas, since low concentrations may indicate chemical, physical or biological pollution, and very high values may indicate eutrophication. Based on the classification proposed by Macedo \& Costa (1978), who considered the values of oxygen saturation rates in order to characterize water quality, the study area can be considered saturated and supersaturated, and the waters classified as zone without pollution. Corroborating the results obtained by Souza et al. (1999), the estuarine system of the São Francisco River can be characterized as free of organic pollution, because, despite the load of debris that the river receives, dissolved oxygen showed high values, possibly due to the large volume of water and current that allows a rapid circulation of water.

The salinity of the estuary at any point depends on the relationship between the volume of salt water and fresh water, the topography, the tidal range and the local climate (McLusky, 1989). The variation gradient of this parameter is very important for algal growth and physiology (Smayda, 1983), interfering in the distribution and abundance of species. In the present study, the levels of salinity, although higher in the rainy season, showed values below 0.5 in both periods, suggesting great fluvial influence, even at the stations closest to the mouth. Similar patterns were reported by Souza et al. (1999). For Knoppers et al. (2005), a saline wedge penetrates up to $10 \mathrm{~km}$ from the mouth and keeps the conditions of surface waters oligohaline. Based on the classification system of water, presented at the symposium in Venice in 1958 (Watanabe, 1997), the São Francisco River estuary can be classified as an oligohaline/limnetic system.

Among the factors that control the growth of Microphytoplanktonic organisms, the availability of dissolved nutrients is very important, especially nitrogen, phosphorus and silicon. The concentration of nutrients such as phosphate, nitrate and silicate is generally greater in the river than in the sea, having a tendency to decrease downstream (Mc Lusky, 1989).

In the present study, the ammonium concentration was significantly higher in the dry season. Nitrite did not differ between the seasons, the recorded values being considered low, and high concentrations of nitrate were recorded in both seasons and were significantly higher in the rainy season. According to Knoppers et al. (2005), in the São Francisco Estuary, nitrogenous elements behave as the main factor limiting primary productivity, especially nitrate as the most important nutrient.

The concentrations of total and dissolved phosphorus were considered low, with higher values in the dry season. In this season, according to Souza et al. (1999), the São Francisco River estuary gets a load of phosphorus-based nutrients from the sugar agribusiness. Greater water transparency during the dry season has conditioned the effective use of these nutrients, resulting in increased Microphytoplankton density and greater specific richness, although the chlorophyll a did not present significant differences with respect to the seasons studied.

In general, as in Medeiros et al. (2011), the mean concentration of dissolved inorganic nutrients, except for silicon, showed a significant depletion in the region of the São Francisco River estuary, confirming the oligotrophic condition of this environment.

The analysis of the micronutrient ratios (nitrogen, phosphorus and silicon) is used to indicate the potential limitation by nitrogen, phosphorus and silicon for the development of Microphytoplankton (Kress et al., 2002). According to Redfield et al. (1963) when the $\mathrm{N}: \mathrm{P}$ molar ratio is less than 16 a possible limitation by nitrogen occurs and when more than 16 the limitation is possibly due to phosphorus. According to Dortch \& Whitledge (1992) and Justic et al. (1995), nitrogen limitation occurs when $N: P$ ratio is less than 10 and the Si:N ratio is greater than 1 ; the limitation by phosphorus is determined when the N:P ratio is higher than 20-30 and the limitation by silicon occurs when the Si:N is greater than 1 and the ratio $\mathrm{Si}: \mathrm{P}$ ratio is greater than 3 .

During the rainy season in the São Francisco River estuary, the N:P ratio mean was high, above 
16, indicating, according to Redfield et al. (1963), the possible limitation of productivity by phosphorus in the rainy season and nitrogen in the dry season. According to Dortch \& Whitledge (1992) and Justic et al. (1995), the limitation by phosphorus occurred only during the rainy season and the limitation by silicon occurred in both seasons. These results indicate the limited availability of phosphorus and silicon in the rainy season and nitrogen and silicon in the dry season.

The N:P ratio in marine and estuarine environments tends to be low and exhibits a relative limitation by nitrogenous forms (Contreras et al., 1996; Nixon et al., 1983). Nitrogen is considered, therefore, the most important nutrient in the regulation of Microphytoplankton production in estuarine systems, and this element is less abundant than phosphorus during peak productivity (Boynton et al., 1982) while in limnetic ecosystems, the limiting factor is phosphorus (Howarth et al., 1988). The atomic N:P ratio of $16: 1$, defined by Redfield, 1958, appears to be an important prerequisite for the development of Microphytoplanktonic organisms, although the different groups of algae have different requirements as to the type of nutrients, being that some species are limited by phosphorus and others by nitrogen (Granéli et al., 1990). Silicon is also considered a limiting factor. However, the rate of recycling of this element in salt water is very high, so it is considered a limiting factor for Microphytoplankton growth only in fresh water (Boney, 1989).

The availability of phosphorus in natural systems significantly affects organic production, thus, the rate of primary production and the increase in Microphytoplankton biomass are influenced by the concentration of this nutrient (Bradford \& Peters, 1987; Flores-Montes et al., 1998). Domestic sewage is a known source of particulate or dissolved phosphorus for coastal environments, where this nutrient is a key factor in controlling pollution since it is produced mainly by discharges and thus becomes easier to control. On the other hand, nitrogen, even when its supply is deficient, can be obtained from atmospheric air by cyanobacteria, making it impossible to control its concentration in water (Branco, 1966; Fonseca et al., 2002).

Among the various pigments, the action of chlorophyll a stands out as it plays a major role in the whole process (Boney, 1989). Therefore, the concentration of chlorophyll a is directly related to the amount of Phytoplankton biomass. Levels of chlorophyll a were relatively low when compared with Passavante \& Koening (1984), Santos-Fernandes et al. (1998), Feitosa et al. (1999), Souza et al. (1999), Grego et al. (2004), Melo-Magalhães et al. (2004) and Melo-Magalhães at al. (2011). The extremely low chlorophyll a concentrations indicate that nutrient uptake by primary production along the mixing zone is minor (Knoppers et al., 2005).

The Microphytoplankton density in the São Francisco River estuary were low compared to results from other studies conducted in estuaries of northeastern Brazil by Sassi (1991), Flores-Montes (1998), Lacerda et al. (2004), Melo-Magalhães et al. (2004).

An atypical behavior was thus ascertained for the phytoplankton of the São Francisco estuary, with great predominance of freshwater species, possibly as a result of low saline values in the estuary. Salinity values under five contributed towards the installation of typically limnetic phytoplankton populations, different from those often observed in other Brazilian estuaries with the presence of a considerable number of marine species (Melo- Magalhães, 2011). The highest densities were recorded in the dry season coinciding with higher values STI. The Bacillariophyta and Chlorophyta were the most representative groups. The most abundant taxa were Aulacoseira ambigua var. ambigua $f$. spiralis, Fragilaria crotonensis and Pediastrum boryanum.

The lowest density values can be assigned to oligotrophic conditions in terms of dissolved nutrients. According to Medeiros (2007; 2011), fertilization and probably the magnitude of primary production at the mouth of São Francisco River were reduced after the completion of the dam in 1995. The river turned into an oligotrophic system with primary production limited by nitrogen and nutrient yields being among the lowest of Brazilian coastal rivers.

This study shows that the levels of chlorophyll a did not follow the seasonal variations observed in the number of Cel.L-1 and of dissolved nutrients. In the dry season, higher cell density and increased levels of nutrients were observed, with the exception of nitrate that was considered higher in the rainy season. The Microphytoplankton species identified are characteristic of waters of low to moderate trophic level and the TSI indicated predominance of oligotrophic conditions in the two seasons in the study area. The general oligotrophic nature of both sources in terms of their nutrient mix and chlorophyll a is, however, one of the remarkable features of the entire São Francisco dispersal system, making it also rather difficult to quantify the behavior of these constituents along the estuarine mixing zone (Knoppers et al., 2005). The oligotrophic conditions of the Estuarine Region of São Francisco River can be possibly attributed to the implementation of dams that caused the retention of nutrients in reservoirs, as well as the Ocean South Equatorial Current, which focuses directly on the coast. 


\section{ACKNOWLEDGMENTS}

The authors thank the São Francisco Hydroelectric Company (CHESF), Instituto do Milênio Estuários CNPq/MCT Project (Process 20.050/2005-1) and INCT-TMCOcean (Process 573.601/2008-9), for the financial support.

\section{REFERENCES}

Agawin, N. S. R. \& Duarte, C. M. 2002. Evidence of direct particle trapping by a tropical seagrass meadow. Estuaries, 25:1205-1209.

Anagnostidis, K. \& Komárek, J. 1990. Modern approach to the classification system of cyanophytes. 3 : Oscillatoriales. Algol. Stud., 50 (53): 327-472.

Boney, A . D. 1989. Microphytoplankton. 2. ed. E. Arnold, London, 118p.

Boynton, W. R.; Kemp, W. M. \& Keefe, C. W. A. 1982. Comparative analysis of nutrients and other factors influencing estuarine Microphytoplankton production. In: Kennedy, V. S. (Ed.). Estuarine comparisons. Academic Press, London. 69-90pp.

Bradford, M. E. \& Peters, R. H. 1987.The relationship between chemically analyzed phosphorus fractions and bioavailable phosphorus. Limnol. Oceanog., 32 (5): 1124-1137.

Branco, S. M. 1966. Estudo das condições sanitárias da Represa Billings, São Paulo. Arquivos Faculdade de Higiene e Saúde Pública da Universidade de São Paulo, 20 (1): 57-86.

Carlson, R. E. 1977. A trophic state index for lakes. Limnol. Oceanog., 2: 361-369.

Cochran, W. G. \& Cox, G. M. Experimental design. 2. ed. J. Wiley, London, 1957. 611p.

Contreras, F.; Castañeda, L.; Torres-Alvarado, R. \& Gutiérrez, F. 1996. Nutrientes em 39 lagunas costeras mexicanas. Rev. Biol.Trop., 44(2): 417-425.

Day Junior, J. W; Hall, C. A. S.; Kemp, W. M. \& YáñezArancibia, A. 1989. Estuarine ecology. J. Wiley, New York, $555 \mathrm{p}$.

Dortch, Q. \& Whitledge, T. E. 1992. Does nitrogen or silicon limit Microphytoplankton production in the Mississippi river plume and nearby regions? Continental Shelf Research, 12: 1293-1309.

Eskinazi-Leça, E. 1967/1969. Shelf off Alagoas and Sergipe (Northeastern Brazil) 3. Diatoms from São Francisco River Mouth. Trab. Oceanogr. Univ. Fed. PE, 9/11:131-192.

Feitosa, F. A. N.; Silva-Cunha, M. G. G.; Passavante, J. Z. O.; Neumann-Leitão, S. \& Lins, I. C. 1999. Estrutura do microfitoplâncton no sistema estuarino do Rio Goiana, Pernambuco, Brasil.
Trab. Oceanogr. Univ. Fed. PE, 27(2): 15-25.

Flores-Montes, M.J.; Macêdo, S.J.; Koening, M. L. \& Correia, I. L. 1998. Variação nictemeral do fitoplâncton e elementos nutrientes no Canal de Santa Cruz, Itamaracá-PE-Brasil. Trab. Oceanogr. Univ. Fed. PE, 26(1): 13-26.

Fonseca, A.; Braga, E. S. \& Eichler, B. B. 2002. Distribuição espacial dos nutrientes inorgânicos dissolvidos e da biomassa fitoplanctônica no sistema pelágico da Lagoa da Conceição, Santa Catarina, Brasil. Atlântica, 24 (2):69-83.

Granéli, E.; Wallström, K.; Larsson, U.; Granéli, W. \& Elmgren, R. 1990. Nutrient limitation of primary production in the Baltic Sea area. Ambio, 19:142-151.

Grasshoff, K.; Ehrhardt, M. \& Kremling, K. 1983. Methods of seawater analysis. 2. ed. Verlag Chemie, New York, 419p.

Grego, C. K. S.; Feitosa, F. A.N.; Honorato, M. S. \& Flores-Montes, M. J. 2004. Distribuição espacial e sazonal da clorofila a fitoplanctônica e hidrologia do Estuário do Rio Timbó (Paulista - PE). Trop. Oceanogr., 32 (2):181-199.

Guiry, M.D. \& Guiry, G.M. 2016. AlgaeBase. Worldwide electronic publication, National University of Ireland, Galway. http://www.algaebase.org; searched on 18 February 2016

Hartmann, C \& Schettini, C. A. F. 1991. Aspectos Hidrológicos na Desembocadura da Laguna dos Patos, RS. Rev. Brasil. Geocien. 21(4):371-377.

Howarth, R. W; Marino, R. \& Lane, J. 1988. Nitrogen fixation in freshwater, estuarine, and marine ecosystems. I. Rates and importance. Limnol. Oceanogr., 33:669-687.

Hustedt, F. 1930. Die Kieselalgen. L. Rabenhorst Kryptogamen-Flora Von Deutschland, Österreichs und der Schweiz, 7:1-920.

Justic, D.; Rabalais, N.N.; Turner, R.E. \& Dortch, Q. 1995.Changes in nutrient structure of riverdominated coastal waters: stoichiometric nutrient balance and its consequences. Estuar. Coast. Shelf Sci., 40: 339-356.

Knoppers, B.; Medeiros, P. R. P.; Souza, W. F. L. \& Jennerjahn, T. 2005. The São Francisco Estuary, Brazil. In: Wangersky, P. (Ed.). The Handbook of environmental chemistry, Water Pollution: estuaries. Springer Verlag, Berlin, 5:1-20.

Kress, N.; Coto, S.L. Bernes, C.L.; Brenner, S. \& Arroyo, G. 2002. Horizontal transport and seasonal distribution of nutrients, dissolved oxygen and chlorophyll-a in Gulf of Nicoya, Costa Rica: a tropical estuary. Cont. Shelf Res., 22:51-66.

Lacerda, S. R.; Koening, M. L.; Neumann-Leitão, S. \& Flores-Montes, M. J. 2004. Microphytoplankton nyctemeral variation at a tropical river estuary 
(Itamaracá - Pernambuco - Brazil). Braz. J. Biol., 64 (1): 81-94.

Macêdo, S.J. \& Costa, K.M.P.1978. Estudos ecológicos da região de Itamaracá. Pernambuco - Brasil. Condições Hidrológicas do Estuário do Rio Botafogo. Ciência e Cultura, 30: 346- 368.

Mafalda-Júnior, P. O.; Oliveira, O. C. \& Souza, C. S. 2002. Análise de padrões espaciais e sazonais na distribuição e abundância do fitoplâncton, zooplâncton e ictioplâncton na região da foz do Rio São Francisco. In: Relatório final do Projeto de Gerenciamento Integrado das Atividades Desenvolvidas em Terra na Bacia do São Francisco Ana/Gef/Pnuma/Oea. Maceió. 2002.

Mc Lusky, D. S. 1989. The estuarine ecosystem. 2.ed. Chapman e Hall, New York, 215 pp.

Medeiros, P. R. P. Knoppers, B. A. Santos Júnior, R. C. Souza, W. F. L. 2007. Aporte fluvial e dispersão de matéria particulada em suspensão na zona costeira do rio São Francisco (SE/AL). Geochimica Brasiliensis, 21(2): 212-231.

Medeiros, P. R. P.; Knoppers, B. A.; Cavalcante, G. H. \& Souza, W. F. L. 2011. Changes in nutrient loads (N, P and Si) in the São Francisco estuary after the construction of dams. Braz. Arch. Biol. Technol., 54 (2): 387-397.

Melo-Magalhães, E. M.; Koening, M. L. \& Sant'Anna, C. L. 2004. Fitoplâncton e variáveis ambientais nos canais do sistema estuarino lagunar Mundau/ Manguaba, Alagoas, Brasil. Hoehnea, 31 (1): 73-86

Melo-Magalhães, E. M.; Koening, M. L. \& Moura, A. N. Complexo estuarino-lagunar Mundaú/Manguaba, Alagoas-Brasil: espécies de algas bioindicadoras de impactos ambientais. 2008. In: Congresso Brasileiro de Ficologia, 11. Simpósio LatinoAmericano Sobre Algas Nocivas, 2006, Itajaí, SC. Anais... Organização da Sociedade Brasileira de Ficologia. (Série Livros, 30), Museu Nacional, Rio de Janeiro.167-185pp.

Melo-Magalhães, E. M.; Moura, A. N.; Medeiros, P.R.P.; Lima, E.L.R. \& Koening, M. L. 2011. Phytoplankton of the São Francisco River estuarine region (Northeastern Brazil): a study of its diversity. Braz. J. Aq. Sc. and Technol. 15(1): 95-105.

Miranda, B. M.; Castro, B. M. \& Kjerve, B. 2002. Princípios de oceanografia física de estuários. EDUSP, São Paulo, $441 \mathrm{pp}$.

Mizuno, T. 1968. Illustrations of freswater plankton of Japan. Osaka: Hoikusha, 351 pp.

Moreno, J. L.; Licea, S. \& Santoyo, H. 1996. Diatomeas del Golfo de California. 1. ed. UABCS, La Paz, $273 \mathrm{pp}$.

Nixon, S .W. \& Pilson, M. E. 1983. Nitrogen in estuarine and coastal marine ecosystems, In: Nitrogen in the
Marine Environment (Carpenter, E. J., Capone, D. G. Eds.). Academic Press, 565-648.

Passavante, J. Z. O. \& Koening, M. L. 1984. Estudo ecológico da região de Itamaracá, Pernambuco -Brasil. XXVI: clorofila "a" e material em suspensão no estuário do rio Botafogo. Trab. Oceanogr. Univ. Fed. PE, 18: 207-230.

Péragallo, H \& Péragallo, M. 1897-1908. Diatommées marines de France et des districtes maritimes voisins. J.Tempere, Paris, $491 \mathrm{pp}$.

Prescott, G. W. 1978. How to know the freshwater algae. Dubuque: Wm. C. Brown, 293 pp.

Pritchard, D. W. 1955. Estuarine circulation pattern. Proceedings of the American Society of Civil Engineers, 81: 1-11.

Round, F. E.; Crawford, R. M. \& Mann, D. G. 1990. The diatoms: biology e morphology of the genera. Cambridge University, Cambridge, 653pp.

Redfield, A.C. 1958. The biological control of chemical factors in the environment. Am. J. Sci., 46: 205-221.

Redfield, A. C.; Ketchum, B. H. \& Richards, F. A. 1963. The influence of organisms on the composition of seawater. In: M. N. Hill (ed.). The sea, 2. Interscience, 26-77pp.

Santos-Fernandes, T. L.; Passavante, J.Z.O.; Koening, M.L. \& Macedo, S.J., 1998. Fitoplâncton do estuário do Rio Jaguaribe, (Itamaracá, Pernambuco, Brasil): biomassa. Trab. Oceanogr. Univ. Fed. PE, 26(2): 1-18.

Sassi, R.1991. Microphytoplankton and environmental factors In the Paraíba do Norte River Estuary, northeastern Brazll: composition, distribution and quantitative remarks. Bol. Inst. Oceanogr. SP, 39(2): 93-115.

Smayda, T. J. 1983. The Microphytoplankton of estuaries. In: Ketchum, M. H. (ed.). Elsevier. Estuaries and Enclose de Seas. Amsterdam. 65-112pp.

Souza, M.R. M.; Silva Cunha, M. G. G. S.; Montes, M. J. F.; Neumann Leitão, S., Porto Neto, F. F.; Moura, M. C. O. \& Chamixaes-Lopez, C. C. B. 1999. Hidrologia e fitoplâncton do Sistema Estuarino do Rio São Francisco, Nordeste do Brasil. Trab. Oceanogr. Univ. Fed. PE, 27(1): 15-31.

Strickland, T. D. H. \& Parsons, T. R. A. 1972. Manual of seawater analysis. Bull. Fish. Res. B. Can., Ottawa.125: 1-205.

Toledo Junior, A. P.; Talarico, M.; Chinex, S. J. \& Agudo, E.E.G., 1983. Aplicação de modelos simplificados para avaliação do processo de eutrofização em lagoas e reservatórios tropicais. In: Congresso Brasileiro de Engenharia Sanitária e Ambiental, 12, Camburiu. Anais... Rio de Janeiro: Sociedade Brasileira de Engenharia Ambiental, 1-34.

Toledo Junior, A. P. 1990. Informe preliminar sobre 
Melo-Magalhães, E.M., et al. (2016). Seasonal biomass of the São Francisco River estuary.

os estudos para obtenção de um índice para avaliação de estado trófico de reservatórios de regiões quentes tropicais. São Paulo: CETESB.
Tomas, C. R. (Ed.). 1997. Identifying marine Microphytoplankton. Academic Press, San Diego, $858 \mathrm{p}$.

Submetido: Março/2014

Revisado: Outubro/2016

Aceito: Novembro/2016 